\section{Mirrors Have a Modest Effect on Human Impulsivity}

\author{
Asami Shinohara ${ }^{1, *}$, Shinya Yamamoto ${ }^{2}$ \\ ${ }^{1}$ Graduate School of Education, Kyoto University, Kyoto 606-8501, Japan \\ ${ }^{2}$ Graduate School of Intercultural Studies, Kobe University, Kobe 657- \\ 8501, Japan \\ "Author for correspondence (a.shinohara92@gmail.com)
}

Human behavior is affected by various social cues. Studies have revealed that cues of being watched affect both social and non-social behavior. A subsequent question is whether one's own image in a mirror has a noticeable effect on behavior and decision-making. Previous studies suggest that people behave in accordance with social desirability in the presence of mirrors; however, the "mirror effect" is still controversial and researchers know little about the mechanism. To further investigate this effect, the current study examined whether mirrors influence impulsivity, a feature that is closely related to decision-making in both social and non-social situations. Participants completed a delay-discounting task in a mirror condition and a no-mirror control condition. We found that relative to the control condition, participant impulsivity increased in the mirror condition. This outcome may have been triggered when the participants' private self-awareness was activated by the mirrors. Thus, mirror-induced self-awareness might have influenced decision-making as a non-social cue in our experiment. Considering the inconsistent results among previous and present studies, the mirror effect might be influenced by the cultural difference or experimental setting. Further investigation is needed to identify how people change their behavior and which aspects of self-awareness are influenced by mirrors.

\section{Keywords}

impulsivity, delay-discounting, mirror effect, selfawareness

\section{Introduction}

Human behavior is influenced by various social cues. Previous studies have revealed that being watched by someone is a social cue that promotes cooperative behavior, such as donating money or picking up garbage, in real-life settings (e.g., Bateson, Nettle, \& Roberts, 2006; Francey \& Bergmüller, 2012). This effect has been replicated in experimental situations with schematic eye drawings (e.g., Haley \& Fessler, 2005; Oda, Niwa, Honma, \& Hiraishi, 2011), although in some cases, no cooperative behavior occurs (e.g., Tane \& Takezawa, 2011). Miyazaki

doi: 10.5178/lebs.2016.46

Received 4 March 2016.

Accepted 22 March 2016.

Published online 18 June 2016.

(C) 2016 by Human Behavior and Evolution Society of Japan
(2013) showed that accuracy increased in a visual search task when participants believed they were being watched by another person. This indicates that even moral-free, non-social behavior and decision-making can be affected by social cues.

What happens with our own reflections? Does one's own image in a mirror have any effect on behavior and decision-making? Previous studies reported that in the presence of mirrors, people were more likely to behave cooperatively (Abbate, Isgrò, Wicklund, \& Boca, 2006; Abbate \& Ruggieri, 2008) or conform to social norms (Diener \& Wallbom, 1976; Wiekens \& Stapel, 2008). Abbate and Ruggieri (2008) found that people were more likely to give money to a beggar when exposed to a mirror. Diener and Wallbom (1976) showed that when people are in a "self-aware" condition, that is, when they are in front of a mirror and listening to their own voice, antinormative behavior (e.g., cheating) decreases compared to when they are in a non-self-aware condition. These results suggest that socially desirable behavior is influenced by mirrors. It is possible that mirror-induced self-awareness affects social decision-making. However, such an effect is still controversial (Niwa, Hiraishi, \& Oda, 2011), and little is known about self-awareness as a mechanism influencing behavior.

To further investigate the mirror effect, this study focused on impulsivity, as measured using a delaydiscounting task. In delay-discounting tasks, participants can choose between two options: an immediate but small reward or a delayed but large reward. Impulsivity is thought to be closely related to decision-making both in social and non-social situations (Baumeister, Vohs, \& Tice, 2007). This study examined how impulsivity changes when participants were exposed to mirrors, thereby identifying how mirrors influence decision-making.

\section{Methods}

(a) Participants

Participants included 31 undergraduate and graduate students (13 men and 18 women, $\left.M_{\text {age }}=21.7, S D=2.9\right)$ at Kobe University, Japan.

\section{(b) Setting}

The experimental room $(313 \mathrm{~cm} \times 300 \mathrm{~cm})$ included a door equipped with non-transparent glass. The room did not have any windows and was occupied by a single participant during each test. Participants were asked to use headphones to minimize interruptions. With the door closed, no one from outside could see inside. The room had two booths separated by a partition. One of the booths (mirror booth) included three mirrors: in front $(52.5 \mathrm{~cm} \times$ $37.0 \mathrm{~cm})$ and on the left and right sides $(151.0 \mathrm{~cm} \times 58.0$ $\mathrm{cm}$ ) of the participant (Figure 1). The mirror directly in front of the participant was set at eye level approximately $60 \mathrm{~cm}$ away. The second booth (no-mirror booth) did not 


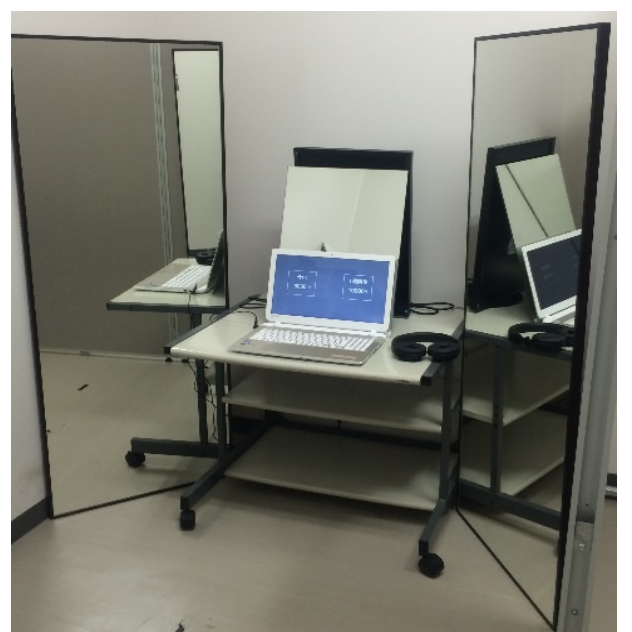

Figure 1. Experimental arrangement for the delay-discounting task in the mirror booth.

have any mirrors. Each booth had a chair and a desk with a laptop computer. Each laptop was used for a decision-making task that appeared on the monitor $(19.5 \mathrm{~cm} \times 34.5 \mathrm{~cm})$; participants were asked to respond using the keyboard.

\section{(c) Procedure}

Before the experiment, participants received instructions and consented to participate. The experimenter explained the monetary reward as hypothetical, but participants were requested to consider it as they would in a real-life setting. Thereafter, participants entered the experimental room alone and began the task at their own pace.

Participants performed a delay-discounting task wherein they were asked to choose one of two payment options: a smaller amount of money obtained right away (immediate money) or a greater amount of money paid after a delay (delayed money). The immediate money option always appeared on the left side of the task monitor, and the delayed money option always appeared on the right. Participants were instructed to press the left-arrow key when they preferred immediate money and the rightarrow key when they preferred delayed money.

To evaluate the amount of immediate money corresponding to a fixed amount of delayed money, we adopted an adjusting-amount procedure (Richards, Mitchell, Wit, \& Seiden, 1997). In every trial, the amount of immediate money was systematically adjusted on the basis of participants' choices in the previous trial: when a participant chose immediate money (or delayed money), the immediate money amount in the next trial decreased (or increased). The amount of delayed money remained fixed (10,000 or 20,000 JPY). The delay periods were one week (immediate money $=6,000 \mathrm{JPY}$ and delayed money $=$ $10,000 \mathrm{JPY}$; immediate money $=12,000 \mathrm{JPY}$ and delayed money $=20,000 \mathrm{JPY}$ ), one month (immediate money $=$ 7,000 JPY and delayed money $=10,000 \mathrm{JPY}$; immediate money $=14,000 \mathrm{JPY}$ and delayed money $=20,000 \mathrm{JPY})$, or one year (immediate money $=8,000 \mathrm{JPY}$ and delayed money $=10,000 \mathrm{JPY}$; immediate money $=16,000 \mathrm{JPY}$ and delayed money $=20,000 \mathrm{JPY}$ ). In total, six combinations of money amount and delay period were used. One test session consisted of 120 trials, 20 trials for each of the six amount-delay period combinations that were mixed and randomized for each participant. PsychoPy v.1.80.03 was used to program the trials.

Participants completed one session for each condition (mirror and control). In the mirror condition, participants performed the task in the mirror booth; in the control condition, participants performed the task in the no-mirror booth. The order of the conditions alternated among participants. Between sessions, participants took a short break outside the experimental room for 3-5 min.

After the experiment, participants completed a questionnaire to identify how much they cared about the mirrors $(1=$ not at all; $7=$ very much $)$.

\section{(d) Data Analysis}

We used an area under the curve (AUC) calculation to identify participants' delay-discount rate (for details about validity and the calculation, see Myerson, Green, \& Warusawitharana, 2001; Reed, Kaplan, \& Brewer, 2012). Theoretically, AUC scores range from 0 (most impulsive) to 1 (least impulsive). For this AUC calculation, the amount of immediate money after the last trial for each amountdelay period combination was used. We obtained two AUC scores (one for 10,000 JPY and the other for 20,000 JPY) for each participant in each condition. Statistical analyses of AUC scores were conducted using R v.3.2.1.

\section{Results}

We conducted a three-way ANOVA (gender [male, female]

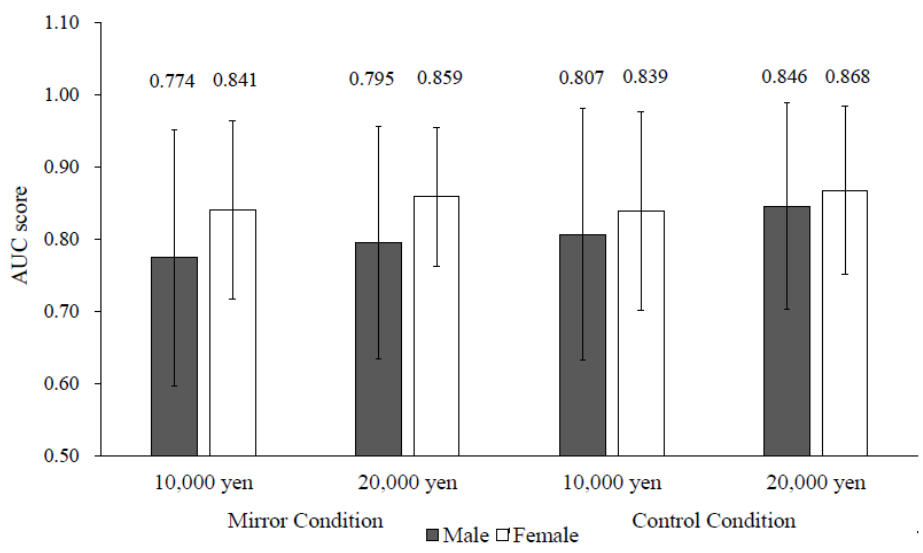

Figure 2. Area under the curve (AUC) score in each condition. Error bars indicate SD. 
$\times$ condition [mirror, control] $\times$ delayed money amount $[10,000 \mathrm{JPY}, 20,000 \mathrm{JPY}])$ on the AUC scores. This analysis revealed a significant main effect of delayed money amount $\left(F=6.88, p=0.014, \eta_{\mathrm{p}}{ }^{2}=0.192\right)$; the AUC of 10,000 JPY (0.819) was significantly lower than that of 20,000 JPY (0.845). We also found a marginally significant main effect of condition $\left(F=3.33, p=0.079, \eta_{\mathrm{p}}{ }^{2}=0.103\right)$; the AUC of the mirror condition (0.822) was lower than that of the control condition (0.842). There was no significant effect of gender $\left(F=0.92, p=0.345, \eta_{\mathrm{p}}{ }^{2}=0.031\right)$ and no significant interactions between gender $\times$ condition $(F$ $\left.=2.35, p=0.136, \eta_{\mathrm{p}}{ }^{2}=0.075\right)$, gender $\times$ delayed money amount $\left(F=0.11, p=0.745, \eta_{\mathrm{p}}^{2}=0.004\right)$, condition $\times$ delayed money amount $\left(F=0.78, p=0.385, \eta_{\mathrm{p}}{ }^{2}=0.026\right)$, or gender $\times$ condition $\times$ delayed money amount $(F=0.04, p=$ $\left.0.836, \eta_{\mathrm{p}}{ }^{2}=0.001\right)$ (Figure 2).

We conducted a correlation analysis to reveal the relationship between the mirror effect, participant impulsivity, and the magnitude of their attention to the mirrors. The mirror effect was calculated by subtracting the AUC score in the control condition from the AUC score in the mirror condition. The magnitude of participant attention was evaluated using questionnaire scores in which participants answered how much they cared about the mirrors during the test. Pearson's product-moment correlation analysis showed no significant relationship $(R$ $=0.150, t=0.819, d f=29, p=0.419$; Figure 3 ). The mirror effect was not related to the participant attentiveness to the mirrors.

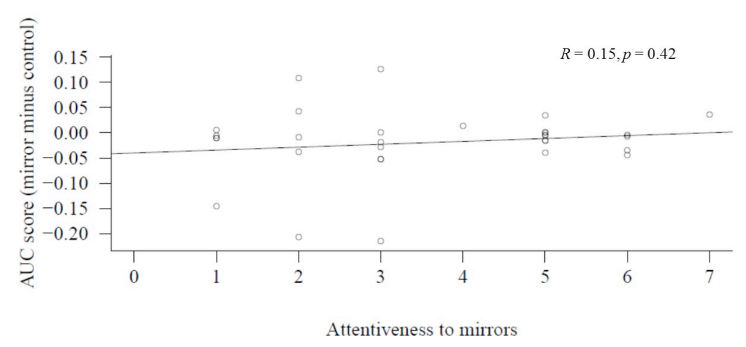

Figure 3. Relationship between attention to mirrors and the effect of mirrors. Attention to the mirrors was measured using a questionnaire. The effect of mirrors was measured as the difference in AUC scores for the mirror and control conditions.

\section{Discussion}

Results revealed that the AUC score increased when the delayed money amount increased. In other words, participants were more impulsive when less delayed money was offered, a result that is consistent with a previous study (Johnson \& Bickel, 2002). This indicates, at least partially, that the experiment was valid, and it succeeded in empirically measuring impulsivity and its anticipated fluctuations within the experimental parameters.

More importantly, concerning the purpose, this study indicated that mirrors have a modest effect on impulsivity. We found the non-significant trend that the AUC score in the mirror condition was lower than the score in the control condition. Although the observed mirror effect was marginal probably due to the relatively small sample size, it might be possible to interpret that mirrors contribute to impulsivity during the delay-discounting task.

Wiekens and Stapel (2008) suggested that mirrors can induce two kinds of self-awareness: public and private. They reported that people conformed to social norms when public self-awareness was activated; conversely, people were more likely to adhere to their own personal opinions when private self-awareness was activated. Considering our own results in line with Wiekens and Stapel's discussion, participants' private self-awareness was possibly activated in the mirror condition in the current study; therefore, participants behaved according to their individual intentions, without any adjustments based on social evaluation. Thus the mirror might have influenced decision-making as a non-social cue. Correlation analysis suggested that the mirror affected participants' decisionmaking without their awareness: even though participants were not paying attention to the mirror, impulsivity scores were higher.

In this study, we investigated the mirror effect on human impulsivity in decision-making. Although previous research has shown that mirror-induced self-awareness promotes socially desirable behavior, we found that selfawareness can increase impulsivity. The mirror effect is still inconsistent, which may be the result of cultural differences (Heine, Takemoto, Moskalenko, Lasaleta, \& Henrich, 2008) or experimental designs. Further investigation is needed to understand the mirror effect in addition to how people change their behavior and which aspects of self-awareness are activated by mirrors.

\section{Acknowledgements}

This study was supported by grants from the Japanese Society of Promotion Sciences (JSPS: Nos. 24700259, 26118509, 15H01619, and 15H05309 to S. Yamamoto). We thank Mr. Tomoya Kawashima for his help in creating the experimental program, Drs. Masako Myowa-Yamakoshi and Eriko Matsumoto, and members of Human Behavior and Evolution Society Japan for their constructive discussion and comments.

\section{References}

Abbate, C. S., Isgrò, A., Wicklund, R. A., \& Boca, S. (2006). A field experiment on perspective-taking, helping, and self-awareness. Basic and Applied Social Psychology, 28, 283-287. (doi: 10.1207/ s15324834basp2803_7)

Abbate, C. S., \& Ruggieri, S. (2008). A beggar, selfawareness and willingness to help. Current Psychology Letters, 24, 98-107.

Bateson, M., Nettle, D., \& Roberts, G. (2006). Cues of being watched enhance cooperation in a real-world setting. Biology Letters, 2, 412-414. (doi: 10.1098/ rsbl.2006.0509)

Baumeister, R. F., Vohs, K. D., \& Tice, D. M. (2007). The strength model of self-control. Current Directions in Psychological Science, 16, 351-355. (doi: 10.1111/ j.1467-8721.2007.00534.x)

Diener, E., \& Wallbom, M. (1976). Effects of selfawareness on antinormative behavior. Journal of Research in Personality, 10, 107-111. 
(doi:10.1016/0092-6566(76)90088-X)

Francey, D., \& Bergmüller, R. (2012). Images of eyes enhance investments in a real-life public good. PLoS ONE, 7, e37397. (doi: 10.1371/journal.pone.0037397)

Haley, K. J., \& Fessler, D. M. T. (2005). Nobody's watching? Subtle cues affect generosity in an anonymous economic game. Evolution and Human Behavior, 26, 245-256. (doi: 10.1016/ j.evolhumbehav.2005.01.002)

Heine, S. J., Takemoto, T., Moskalenko, S., Lasaleta, J., \& Henrich, J. (2008). Mirrors in the head: Cultural variation in objective self-awareness. Personality and Social Psychology Bulletin, 34, 879-887. (doi: 10.1177/0146167208316921)

Johnson, M. W., \& Bickel, W. K. (2002). Within-subject comparison of real and hypothetical money rewards in delay discounting. Journal of the Experimental Analysis of Behavior, 77, 129-146. (doi: 10.1901/ jeab.2002.77-129)

Miyazaki, Y. (2013). Increasing visual search accuracy by being watched. PLoS ONE, 8, e53500. (doi: 10.1371/ journal.pone.0053500)

Myerson, J., Green, L., \& Warusawitharana, M. (2001). Area under the curve as a measure of discounting. Journal of the Experimental Analysis of Behavior, 76, 235-243. (doi: 10.1901/jeab.2001.76-235)

Niwa, Y., Hiraishi, K., \& Oda, R. (2011). A mirror has no effect on giving in the dictator game. Letters on Evolutionary Behavioral Science, 2, 16-19. (doi: 10.5178/lebs.2011.13)

Oda, R., Niwa, Y., Honma, A., \& Hiraishi, K. (2011). An eye-like painting enhances the expectation of a good reputation. Evolution and Human Behavior, 32, 166171. (doi: 10.1016/j.evolhumbehav.2010.11.002)

Reed, D. D., Kaplan, B. A., \& Brewer, A. T. (2012). A tutorial on the use of Excel 2010 and Excel for Mac 2011 for conducting delay-discounting analyses. Journal of Applied Behavioral Analysis, 45, 375-386. (doi: 10.1901/jaba.2012.45-375)

Richards, J. B., Mitchell, S. H., Wit, H. D., \& Seiden, L. S. (1997). Determination of discount functions in rats with an adjusting-amount procedure. Journal of the Experimental Analysis of Behavior, 67, 353-366. (doi: 10.1901/jeab.1997.67-353)

Tane, K., \& Takezawa, M. (2011). Perception of human face does not induce cooperation in darkness. Letters on Evolutionary Behavioral Science, 2, 24-27. (doi: 10.5178/lebs.2011.15)

Wiekens, C. J., \& Stapel, D. A. (2008). The mirror and I: When private opinions are in conflict with public norms. Journal of Experimental Social Psychology, 44, 1160-1166. (doi: 10.1016/j.jesp.2008.02.005) 